\title{
La communication engageante au service de l'éducation inclusive : Etude expérimentale
}

\section{Engaging Communication for Inclusive Education: Experimental Study}

\author{
Lahcen Seddik ${ }^{1, *}$ \\ ${ }^{1}$ Laboratoire des Sciences Humaines Appliquées, USMBA, 30000 Fès, Maroc
}

\begin{abstract}
Résumé. L'éducation inclusive des enfants en situation d'handicap implique la remise en question du paradigme éducationnel traditionnel. Ceci nécessite la transformation radicale du système scolaire en place de façon à ce que tous les enfants aient une place au sein de l'école de leur quartier de résidence. Ainsi la révision des curricula et des dispositifs de formation initiale et qualifiante s'impose d'emblée. Par ailleurs, l'implication et l'engagement des enseignants constituent-ils un des défis majeurs face à l'implantation de ce nouveau paradigme. Leurs résistances à un tel changement constituent un facteur de risque d'échec de l'éducation inclusive. D'où la nécessité de mener une campagne d'information et de communication au sein des écoles pour amener les enseignants à s'y engager librement et sans aucune contrainte. Cet article a pour objet la présentation des résultats d'une recherche expérimentale sur l'incidence de la communication engageante sur l'intention comportementale des enseignants au regard de l'éducation inclusive. L'objectif en est de comparer l'efficacité d'une démarche de communication engageante à celle d'engagement et à celle de communication persuasive. Les résultats de cette expérimentation montrent que les participants, ayant été soumis à une démarche de communication engageante, ont exprimé une intention plus forte d'intégrer les élèves en situation de handicap dans leur classe, en comparaison avec ceux qui ont été soumis à une démarche d'engagement ou de persuasion.
\end{abstract}

\begin{abstract}
Inclusive education for children with disabilities involves reconsidering the traditional education paradigm. This requires a radical transformation of the existing school system so that all children have a place in the local school. It is therefore necessary to revise the curricula as well as the initial and qualifying education systems from the outset. In addition, the involvement and commitment of teachers is one of the major challenges facing the implementation of this new paradigm. Their resistance to such a change is a risk factor for the failure of inclusive education. Hence the need to conduct an information and communication campaign within schools to encourage teachers to get engaged in it freely and without any constraint.
\end{abstract}

*Corresponding author: lahcen.seddik1@usmba.ac.ma 
The purpose of this article is to show the results of an experimental research on the impact of engaging communication on the behavioural intention of teachers with regard to inclusive education. The aim is to compare the effectiveness of an engaging communication process with that of engagement and that of persuasive communication. The results of this experiment show that the participants with whom an engaging communication process was carried out expressed a stronger intention to integrate students with disabilities into their class, compared to those who were subjected to a process of engagement or persuasion.

\section{Introduction}

Vers la fin des années 1990, le Maroc met en œuvre une stratégie nationale visant l'intégration scolaire des enfants en situation d'handicap. Cette stratégie consiste à créer des classes d'intégration scolaire (ou clis) au sein des écoles primaires à l'échelon national. Il s'agit de structures spécialisées dans l'éducation et l'intégration scolaire du handicap. Cette formule d'intégration scolaire n'impose pas aux écoles de véritables changements. Elle implique seulement quelques aménagements en termes de rythmes scolaires, de locaux et de matériels didactiques. De surcroît, on y accueille exclusivement les enfants porteurs d'un handicap léger ou moyen, c'est-à-dire ceux qui sont capables de s'adapter au système scolaire en place. Certes, cette modalité d'intégration a permis de rompre temporairement l'isolement d'un certain nombre d'élèves en situation d'handicap, mais force est de constater qu'elle présente des limites et des défauts incontournables. Ainsi, cette structure, censée préparer l'intégration scolaire totale des enfants en situation d'handicap, s'est transformée, au fil du temps, en "ghetto ». En plus, les acquis scolaires et sociaux des enfants en situation d'handicap, inscrits dans ces clis, restent en deçà des attentes et des aspirations de leurs parents et de leurs enseignants. En conséquence, le taux des élèves en situation d'handicap ayant pu franchir le primaire vers le cycle collégial est quasiment négligeable. Leur retour, après quelques années passées en clis, dans les milieux spécialisés est inévitable. Ainsi, au lieu de constituer un premier pas dans un cursus scolaire plus long (primaire, collège, lycée, université), la clis s'est métamorphosée en une micro-institution spécialisée au sein d'une école ordinaire. Chose qui a généré une stigmatisation aussi bien de ses usagers que de ses enseignants. Une telle réalité a été, peut-être, générée par plusieurs facteurs, entre autres, les résistances au changement, les représentations sociales de l'handicap, les dysfonctionnements organisationnels, le manque de préparation des clis, les problèmes liés au mode de communication, le manque de formation des enseignants, ... etc.

Aujourd'hui, les autorités éducatives marocaines décident d'asseoir une école inclusive, et ce en application de la Convention internationale relative aux droits des personnes handicapées, ratifiée par le Maroc en 2009. L'école inclusive vise l'intégration pédagogique de tous les enfants en situation d'handicap ou de difficulté d'adaptation, et ce peu importe la nature et la sévérité de leur handicap. Cette nouvelle réforme pédagogique nécessite une préparation de grande envergure : réforme des curricula et programmes scolaires, réforme des dispositifs de formation des enseignants, des inspecteurs et des cadres administratifs, réforme des rythmes scolaires et des modes d'évaluation...etc. En plus, elle implique l'organisation des formations continues et des campagnes de sensibilisation et d'information au profit des acteurs de l'école et de la communauté locale toute entière.

Par ailleurs, cette réforme est susceptible d'engendrer de fortes résistances et réticences de la part des enseignants, dans la mesure où leur engagement et leur implication effective dans l'éducation inclusive vont les contraindre à multiplier leurs efforts. Ainsi, au lieu de gérer les apprentissages d'un groupe, supposé "homogène », ils vont travailler avec un groupe d'élèves hétérogène. D'où l'intérêt de différencier et d'individualiser les 
apprentissages, et partant, de travailler avec des projets pédagogiques individualisés. Cela implique un travail d'équipe. Dans cette logique, l'enseignant n'est plus un simple pédagogue et didacticien qui prépare, gère et évalue les savoirs de la discipline. Il est plutôt un membre d'une équipe pluridisciplinaire, qui établit et met en œuvre, pour chaque élève, un bilan multidimensionnel et un projet médico-psycho-socio-pédagogique. Dans l'éducation inclusive, l'accent est mis plus sur les besoins, les intérêts, le potentiel et les rythmes individuels de chaque élève que sur les contenus scolaires. En effet, il s'agit, pour l'enseignant, d'une tâche beaucoup plus complexe que ce qu'il a l'habitude de réaliser dans sa classe dite "normale ». La mise en œuvre de l'éducation inclusive, dans le contexte scolaire marocain, nécessite à priori une préparation du terrain dont l'un des aspects importants consiste à convaincre les différents acteurs de l'école à s'y engager en actes.

Cette étude expérimentale a pour objet l'étude des effets de la communication engageante en matière de changement de comportement des enseignants vis-à-vis de l'éducation inclusive. L'objectif en est de comparer l'efficacité pratique d'une démarche de communication engageante à celle d'engagement et à celle de communication persuasive. Son utilité pratique réside, semble-t-il, dans le fait de mettre à la disposition des décideurs un outil, aussi efficace que possible, à même de convaincre les enseignants réticents de s'engager dans l'éducation inclusive.

\section{Cadre conceptuel}

\subsection{1 L'éducation inclusive}

L'éducation inclusive est un nouveau paradigme éducationnel qui consiste à passer de la centration sur les difficultés de l'élève à la centration sur les conditions d'enseignementapprentissage [1]. Par opposition à l'intégration scolaire qui vise la rupture de l'isolement des enfants en situation d'handicap léger ou moyen, l'éducation inclusive a pour finalité l'intégration pédagogique, à temps plein, de tous les enfants en situation d'handicap ou en difficulté d'apprentissage ou d'adaptation, sans aucune restriction [2]. Elle repose sur le postulat selon lequel tous les acteurs de l'école s'engagent et s'impliquent dans le processus d'inclusion [3]. Ainsi, elle suppose le passage d'un service spécialisé à une collaboration de l'ensemble des intervenants, d'un système éducatif standardisé à une école qui prend en compte les caractéristiques de l'ensemble des élèves, d'un parcours construit pour les élèves dits valides à un système pour tous [3]. De surcroit, elle implique, d'une part, la participation optimale de chaque élève à toutes les activités d'apprentissage de son groupe classe, et, d'autre part, sa participation à la vie sociale de l'école.

Ce nouveau paradigme nécessite la transformation radicale du système d'éducation de manière à développer sa capacité à répondre aux besoins de tous les apprenants [4], [3], [5] . Dans cette même perspective, Armstrong insiste sur le fait que l'éducation inclusive implique une double transformation : d'un côté, il s'agit de transformer les écoles de telle sorte qu'elles soient ouvertes pour tous sans restriction; de l'autre côté, il faut changer, les pratiques pédagogiques pour répondre aux besoins de tous [6]. Or, les pratiques pédagogiques traditionnelles sont ancrées dans les conceptions des enseignants [3]. Chose qui pourrait impacter les attitudes et les comportements des enseignants face à l'implantation de ce nouveau paradigme. Le changement de ces dites pratiques passe nécessairement par la formation initiale et continue des enseignants. De surcroit, la diversité, générée par l'inclusion, suppose un travail de transformation et de modification du cursus type en place.

Par ailleurs, l'éducation inclusive n'implique pas pour autant la négation du handicap. Au contraire, le handicap constitue une donnée, entre autres, que l'école inclusive prend en compte, traite et, par conséquent, gère [7]. L'indifférence à la différence est un principe banni 
par ce nouveau paradigme. Ainsi, la diversité y est considérée comme une richesse et non un obstacle. D'où l'individualisation du processus d'enseignement apprentissage réalisée à l'aide de stratégies d'enseignement et de modes d'évaluation diversifiés et par la valorisation du caractère unique de chaque apprenant [2].

\subsection{La communication engageante}

En psychologie sociale, le paradigme de la communication engageante s'appuie sur deux autres paradigmes à savoir le paradigme de la soumission librement consentie (théorie de l'engagement) et le paradigme de la communication persuasive [8].

Le paradigme de la soumission librement consentie procède à l'examen des techniques psychosociales d'influence à même d'amener autrui à modifier librement ses attitudes et ses comportements en réalisant des actes préparatoires et des actes d'engagement [8], [9]. L'engagement renvoie, dans une situation donnée, "aux conditions dans lesquelles on attribue l'acte à celui qui l'a réalisé, et non pas aux facteurs externes » [10, p. 24]. Ainsi, le principe de base de la soumission librement consentie consiste à amener l'individu à réaliser un acte sans se sentir contraint de le faire [11]. Parmi les techniques d'influence les plus étudiées, on peut citer la technique du «pied-dans-la porte» qui consiste à obtenir peu (acte préparatoire) avant de demander davantage (requête finale), et ce dans un contexte de liberté [11, p. 41]. C'est l'une des techniques de manipulation utilisées dans le marketing. Ainsi, les travaux de recherche, réalisés dans le cadre de ce paradigme, montrent que le changement du comportement est tributaire de la réalisation, par les sujets, des actes préparatoires [12].

Le paradigme de la communication persuasive, quant à lui, étudie les procédures et les techniques de persuasion par le discours (texte, affiche, image...). Ainsi les travaux sur la persuasion essayent de répondre à la question : «qui dit quoi, à qui, dans quel canal et avec quel effet? » [11, p. 56]. Ainsi, les variables étudiées sont les caractéristiques de la source (crédible vs non crédible), la construction du message (arguments forts vs faibles; argumentation unilatérale vs bilatérale ; conclusion explicite vs implicite) et le contexte de diffusion du message (agréable vs désagréable ; choix libre vs contrainte ; appel à la peur vs non appel à la peur) [8], [12], [13]. Grosso modo, les chercheurs en matière de persuasion pensent que le changement des comportements des individus passe inexorablement par le changement de leurs attitudes [11]. Néanmoins, les recherches en ce domaine montrent que la communication persuasive est susceptible d'entrainer le changement des attitudes, mais son incidence sur le comportement des sujets est quasi-négligeable [12].

Pour ce qui concerne la communication engageante, elle «repose sur la réalisation préalable d'un ou de plusieurs acte(s) préparatoire(s) engageant(s) associée à une argumentation persuasive allant dans le même sens que l'acte précédemment réalisé » [14, p.45]. Autrement dit, avant de procéder, par exemple, à une campagne d'information et de communication visant le changement d'attitudes et de comportements des personnes vis-àvis d'une question donnée, il faudrait a priori les amener à réaliser un acte préparatoire, peu couteux, mais fortement engageant. Les actes préparatoires (ou «mini-actes»), qui précèdent la lecture et l'analyse du message persuasif, induisent les sujets à réaliser des traitements cognitifs et affectifs à même d'engendrer une persuasion plus forte [15]. Ainsi, la communication engageante essaye de répondre à la question suivante : "qui dit quoi, à qui, dans quel canal, avec quel soubassement comportemental ?» [11, p. 57], [13, p. 287]. Le soubassement comportemental est favorisé par la réalisation, par le sujet, de l'acte préparatoire. Ce dit acte préparatoire doit obéir à un certain nombre de conditions d'efficacité [8], [9], [15] :

- il doit s'inscrire dans le même champ d'action que le comportement attendu.

- il doit être réalisé dans un contexte d'engagement (libre choix, absence de promesse de récompense ou de menace de punition). 
- il doit avoir un certain coût et être effectivement réalisé.

- la personne qui réalise cet acte doit l'expliquer par des facteurs internes, tels que ses goûts, ses convictions et ses attitudes.

La différence entre la communication engageante et la communication persuasive réside dans le fait que la communication engageante permet au sujet cible d'avoir un statut d'acteur, et non seulement de récepteur passif [8]. Ainsi, l'acte préparatoire, réalisé librement et sans aucune contrainte ou promesse, amène le sujet à s'engager fortement, en tant qu'acteur, dans un processus dynamique de changement attitudinal et comportemental. La communication engageante insiste alors sur la création des conditions favorables pour qu'un acte soit réalisé [16]. Elle peut être utilisée alors comme une méthode ou stratégie de changement de comportement social des individus [12]. Par ailleurs, les recherches expérimentales et recherches-actions, menées dans le cadre de ce paradigme, montrent qu'effectivement la communication engageante entraine un changement, non seulement d'attitudes, mais également de comportements des sujets.

\section{Cadre méthodologique}

\subsection{Problématique}

Au Maroc, malgré les efforts déployés par le ministère de l'éducation nationale (stratégies d'intervention, campagnes d'information, formation des enseignants, ...) les taux de scolarisation normale des enfants en situation d'handicap sont significativement faibles. Les facteurs déterminants d'une telle situation sont certes multiples, mais force est de constater que les stratégies de communication utilisées par le ministère (circulaires, notes ministérielles, réunions, ...etc.), afin d'amener les enseignants à s'impliquer dans le processus d'intégration des enfants en situation d'handicap, sont stériles voire inutiles. Elles renforcent leurs résistances et réticences au regard d'une telle intégration. Ces résistances s'avèrent alors normales tant que la démarche de persuasion utilisée est loin d'être fortement engageante. Informer et sensibiliser est une stratégie qui peut influencer les attitudes des gens mais pas forcément leurs actes [12]. Un enseignant pourrait, par exemple, être convaincu de l'intérêt d'intégrer à l'école les enfants en situation d'handicap sans pour autant accepter de le faire dans sa propre classe. Ainsi, une étude longitudinale sur les attitudes des enseignants envers l'intégration scolaire des handicapés, montrent que $66 \%$ des enseignants interrogés sont d'accord avec l'idée et le principe de l'intégration scolaire, mais que seulement le tiers des enseignants de ce groupe sont d'accord de le faire dans leur propre classe [17].

Notre recherche, s'inscrivant dans le paradigme de la communication engageante, essaye de répondre au questionnement suivant : l'intention de s'engager (ou non) dans l'éducation inclusive dépend-t-elle, ou non, de la démarche de communication adoptée ? La démarche de communication engageante peut-elle induire les enseignants à s'engager dans l'éducation inclusive? La communication engageante est-elle plus efficace que la communication persuasive et la soumission librement consentie?

\subsection{Hypothèse de travail}

Eu égard au paradigme choisi, notre hypothèse peut être formulée ainsi : les participants ayant réalisé un acte préparatoire fortement engageant, et par la suite, lu un texte à visée persuasive (condition de communication engageante) exprimeraient une intention plus forte d'intégrer dans leur classe les élèves en situation d'handicap, que les participants ayant seulement réalisé l'acte préparatoire (condition d'engagement) et que les participants ayant seulement lu le texte persuasif ( condition de persuasion). 


\subsection{Echantillons}

Les groupes expérimentaux ont été constitués à partir des listes de trois différentes filières de la licence professionnelle en enseignement au sein de l'ENS de Fès. Il s'agit des licences professionnelles en Psychologie et Apprentissage (LPPA), en Education Physique et Sportive (LPEPS) et en Technologies Educatives et Développement Multimédia(TEDEMU). L'effectif global des participants à l'expérimentation est 69, dont 43 garçons et 26 filles, à raison de 23 participants par groupe.

\subsection{Démarche expérimentale}

La démarche utilisée consiste à soumettre chacun des trois groupes à une condition particulière. Ainsi, le premier groupe (LPPA) a été soumis à une condition d'engagement. Dans cette condition, les participants sont d'abord amenés à réaliser un acte préparatoire qui consiste à rédiger une lettre, entre 15 et 20 lignes, à $\mathrm{Mr}$ le ministre de l'éducation nationale dans laquelle ils l'exhortent à prendre des mesures nécessaires pour promouvoir l'éducation inclusive des enfants en situation d'handicap. Après avoir écrit cette lettre (acte préparatoire), on leur demande de signer un engagement dans lequel ils sont invités à exprimer, librement et sans aucune contrainte, leur intention d'intégrer dans leur classe (ou de rejeter) les élèves en situation d'handicap.

Le second groupe (LPEPS), quant à lui, est invité, dans un premier temps, à lire un extrait (en arabe) de la Déclaration de Salamanque relative aux principes, aux politiques et aux pratiques en matière d'éducation et d'enseignement des enfants à besoins spécifiques (7-10 juin 1994). Ce texte détermine le principe (éthique et pédagogique) fondateur de l'éducation inclusive ainsi que ses objectifs, ses stratégies et ses ressources. Il relate également les expériences, les pratiques et les différents systèmes de prise en charge pédagogique des enfants en situation de handicap. En guise de conclusion, ce texte recommande aux pays en voie de développement d'adopter l'éducation inclusive vue ses avantages économiques, sociaux et éducatifs. Après avoir lu ce texte, les participants ont été invités à signer l'engagement précité.

Le troisième groupe (TEDEMU), lui, a été soumis à une condition de communication engageante. D'abord, on leur a demandé de réaliser l'acte préparatoire susmentionné (rédiger la lettre). Ensuite, ils ont été amenés à lire le texte persuasif précité (extrait de la Déclaration de Salamanque). Enfin, on leur a demandé de signer le même engagement que les deux premiers groupes.

Concernant le traitement statistique des données de l'expérimentation, on a utilisé le logiciel XLSTAT (version d'essai disponible sur le Web). On a procédé au test paramétrique d'association de deux variables qualitatives (test sur tableaux de contingence), en l'occurrence le khi-2, pour vérifier dans quelle mesure l'intention d'intégrer (ou de rejeter) les élèves en situation de handicap dépendent de la démarche de communication utilisée. La $\mathrm{p}$-value calculée doit être inférieure au niveau de signification alpha $=0,05$ pour rejeter l'hypothèse nulle et retenir l'hypothèse alternative selon laquelle il existe un lien entre les deux variables en question.

\section{Analyse et discussion des résultats}

\subsection{Analyse des résultats}

D'après le tableau de contingence ci-dessous, les participants ayant été soumis à une condition de communication engageante sont significativement plus nombreux $(56,52 \%)$ à 
exprimer une intention forte d'intégrer -dans leur classe -les élèves en situation d'handicap, que les participants ayant été soumis à une condition de communication persuasive $(8,7 \%)$, et que les participants ayant été soumis à une condition d'engagement $(17,39 \%)$.

Table 1. Tableau de contingence en $\%$.

\begin{tabular}{|c|c|c|c|}
\hline \multirow{2}{*}{$\begin{array}{c}\text { Intention } \\
\text { comportementale }\end{array}$} & \multicolumn{3}{|c|}{ Condition de communication } \\
\cline { 2 - 4 } & $\begin{array}{c}\text { Communication } \\
\text { engageante }\end{array}$ & $\begin{array}{c}\text { Engagement par } \\
\text { les actes }\end{array}$ & Persuasion \\
\hline Moyenne & 43,48 & 69,57 & 43,48 \\
\hline Forte & 56,52 & 17,39 & 8,70 \\
\hline Nulle & 0,00 & 13,04 & 47,83 \\
\hline Total & 100,00 & 100,00 & 100,00 \\
\hline
\end{tabular}

On peut d'ores et déjà, en regardant ces données, affirmer que la variable « intention comportementale » dépend de la variable « condition de communication». Pour vérifier la véracité de ce résultat, on a procédé au test du khi-2.

Table 2. Test d'indépendance entre les lignes et les colonnes $\left(\mathrm{Khi}^{2}\right)$.

\begin{tabular}{|c|c|}
\hline $\mathrm{Khi}^{2}$ (Valeur observée) & 26,699 \\
\hline $\mathrm{Khi}^{2}$ (Valeur critique) & 9,488 \\
\hline DDL & 4 \\
\hline p-value & $<0,0001$ \\
\hline Alpha & 0,05 \\
\hline
\end{tabular}

Ainsi, la $p$-value calculée est inférieure au niveau de signification alpha $=0,05$; d'où le rejet de l'hypothèse nulle $(\mathrm{H} 0)$ et l'acceptation de l'hypothèse alternative $(\mathrm{Ha})$ selon laquelle il existe un lien entre les deux variables qualitatives. On en conclue que l'intention d'intégrer (ou de rejeter) les élèves en situation d'handicap dépend, en grande partie, de la démarche de communication utilisée. En effet, les participants ayant rédigé la lettre à Mr le ministre (acte préparatoire) et, par la suite, ont lu l'extrait de la déclaration de Salamanque (message persuasif) (condition de communication engageante) expriment une intention forte d'intégrer -dans leur classe- les élèves en situation d'handicap, que les participants ayant seulement écrit la lettre (condition d'engagement) et que les participants ayant seulement lu l'extrait de ladite déclaration (condition de persuasion). Chose qui tend à confirmer l'hypothèse de cette recherche.

\subsection{Discussion des résultats}

Certes, il n'existe pas de travaux de recherche associant la variable intention comportementale au regard de l'éducation inclusive et la communication engageante, mais force est de constater que le résultat de cette étude expérimentale tend à confirmer les résultats des recherches antérieures en matière de communication engageante. Ainsi, l'étude expérimentale, menée par Girandola et ses collaborateurs [12], montre que les participants ayant accepté de porter le badge "défi pour la terre" (acte préparatoire) et ayant lu le message persuasif (un texte de 20 lignes portant sur les avantages du recyclage) (condition de communication engageante) expriment une attitude plus positive envers le recyclage et une intention plus forte de recycler les déchets, que les participants ayant seulement réalisé l'acte préparatoire (condition d'engagement) et que les participants ayant seulement lu le message ( condition de persuasion). De même, les études expérimentales sur l'activité physique des étudiants montrent que les étudiants expriment davantage l'intention de pratiquer une activité physique après avoir réalisé un acte préparatoire (rédiger un texte en faveur d'une pratique physique) et lu un message persuasif sur les bienfaits d'une activité physique (condition communication engageante), qu'après avoir seulement lu le message (communication persuasive) [8]. Dans cette même optique, l'étude expérimentale sur les 
économies d'énergies montre que les participants ayant rédigé, pendant 10 minutes, un message en faveur des économies d'énergies (acte préparatoire) et ayant, par la suite, lu un texte d'une quinzaine de lignes, portant sur la nécessité d'une meilleure maitrise énergétique (message persuasif) (condition de communication engageante), ont une attitude plus favorable vis-à-vis d'une augmentation des impôts visant à soutenir une politique d'économie énergétique et expriment une plus forte intention de payer plus d'impôts que dans la condition d'engagement ( acte préparatoire seul) et que dans la condition de persuasion (message seul) [12]. La recherche-action sur le don d'organes montre que les participants ayant répondu à quelques questions sur le don d'organes (acte préparatoire) avant de lire un message argumentatif sur le même sujet (message persuasif) (condition de communication engageante) sont significativement plus nombreux à signer une carte de donneur d'organes que ceux qui ont seulement lu le message argumentatif (condition de persuasion) [12]. Dans la même perspective, la recherche-action sur le vote des Suisses montre que le taux de participation au vote est significativement plus élevé dans les conditions de communication engageante, que dans la condition de communication de persuasion [12].

Toutes ces recherches montrent que la communication engageante, par opposition à la communication persuasive, est à même de changer aussi bien les attitudes que les comportements des gens vis-à-vis d'une question donnée. La communication persuasive, à elle seule, peut induire des changements d'idées et d'attitudes et non le changement de comportements. La preuve en est les résultats d'un certain nombre de recherches qui ont montré que la modification des idées et des attitudes n'entraine pas automatiquement le changement de comportement des gens. Ainsi, l'étude longitudinale sur la prévention des jeunes du tabac [8], la méta-analyse portant sur 350 campagnes de prévention du sida [8] et la méta-analyse de 400 articles et rapports relatifs aux campagnes anti-alcool [12] montrent que ces campagnes contribuent au changement des connaissances des concernés et non au changement de leurs comportements.

\section{Conclusion}

Cette recherche a eu pour objet l'analyse de l'incidence de la communication engageante sur l'intention comportementale des enseignants au regard de l'éducation inclusive des enfants en situation d'handicap. Il s'agit d'une première étude, à caractère expérimental, à tenter d'associer la communication engageante et l'éducation inclusive. Ses résultats ont certes montré que les participants ayant été soumis à une démarche de communication engageante expriment une intention forte de s'engager en actes dans l'éducation inclusive, en comparaison avec ceux qui ont été soumis à une démarche d'engagement ou de persuasion, mais force est de constater que la taille de l'échantillon, ses caractéristiques ainsi que le champ de l'expérimentation ne nous permettent pas de procéder à une généralisation de ces résultats. Encore faut-il réaliser une telle expérimentation au sein des écoles, avec un échantillon beaucoup plus important et avec une démarche et des outils diversifiée. Néanmoins, l'on peut affirmer que la communication engageante peut être utilisée comme une stratégie de persuasion à même d'amener les enseignants à s'engager dans l'éducation inclusive.

\section{Références}

1. F. James, Promouvoir l'inclusion, stratégies pour la classe. Dans B. Belmont et A. Vérillon (dir.), Diversité et handicap à l'école, quelles pratiques éducatives pour tous ?, 100-117, (INRP \& CTNERHI, Paris, 2003). 
2. R. Vienneau, Impacts de l'inclusion scolaire sur l'apprentissage et sur le développement social. Dans N. Rousseau et S. Belanger (dir.), La pédagogie de l'inclusion, 127-152, (Press de l'Université du Québec, Québec, 2004).

3. D. Curchod-Ruedi, S. Ramel, P. Bonvin, O. Albanese et P.A. Doudin, De l'intégration à l'inclusion scolaire : implication des enseignants et importance du soutien social AEJDR, 7, 135-147 (2013).

4. R. Vienneau, De l'intégration scolaire à une véritable pédagogie de l'inclusion. Dans C. Dionne et N. Rousseau (dir.), Transformations des pratiques éducatives ; la recherche sur l'inclusion, 7-32, (Press de l'université du Québec, Québec, 2006).

5. S. Thomazet, L'intégration a des limites, pas l'école inclusive !, RSE, 34 (1), 123-139 (2008).

6. F. Armstrong, Les paradoxes de l'éducation inclusive en Angleterre. Dans M. Chauvière et E. Plaisance (dir.), L'école face aux handicaps, éducation spéciale ou éducation intégrative, 117-132, (Puf, Paris, 2005).

7. M. F. Berthe-Denoux et V. Leoni, Le regard des enfants valides. Dans M. Chauvière et E. Plaisance (dir.), L'école face aux handicaps, éducation spéciale ou éducation intégrative, 189-203, (Puf, Paris, 2005).

8. F. Girandola et R. Joule, La communication engageante, REPS, 2, $41-51$ (2008).

9. C. Eyssartier, R. Joule et C. Guimelli, Effets comportementaux et cognitifs de l'engagement dans un acte préparatoire activant un élément central versus périphérique de la représentation du don d'organes, PF, 52, 499-517 (2007).

10. F. Bernard et R. Joule, Lien, sens et action : vers une communication engageante, CO, 24, 333-345 (2004).

11. F. Michelik, La communication engageante : effets sur les dimensions cognitives et comportementales, Thèse de doctorat, Université de Franche-Comté (2011). https://tel.archives-ouvertes.fr/tel-00840255

12. F. Girandola et R. Joule, La communication engageante : aspects théoriques, résultats et perspectives, AP, 112, 115-14 (2012).

13. A. Zbinden, L. Souchet, F. Girandola et G. Bourg, Communication engageante et représentations sociales : une application en faveur de la protection de l'environnement et du recyclage, PP, 17, 285-299 (2011).

14. C. Solanas, J.C. Colavolpe et A. Geissler, La communication engageante au service du don de cornées. Dans A. Fluckiger (dir.), Emouvoir et persuader pour promouvoir le don d'organes? L'efficacité entre éthique et droit, 43-54 (Schulthess, 2010).

15. D. Courbet, M. P. Fourquet-Courbet, F. Bernard et R.V. Joule, Communication persuasive et Communication engageante pour la santé. Favoriser des comportements sains avec les médias, Internet et les serious games. Dans N. Blanc (dir.), Publicité et Santé : des liaisons dangereuses ? Le point de vue de la psychologie, 1-15, (Press, Paris, 2013).

16. F. Bernard, Communication engageante, environnement et écocitoyenneté : un exemple des « migrations conceptuelles » entre SIC et psychologie sociale, CO, 31, 27-41 (2007).

17. L. Lamontagne-Muller, Les attitudes envers l'intégration scolaire d'élèves en situation de handicap et l'attitude envers les personnes en situation de handicap. Les rôles des modèles individuels et sociaux du handicap dans le processus de persuasion, Thèse de doctorat, Université de Fribourg- Suisse (Fribourg, 2007).

https://core.ac.uk/download/pdf/20641605.pdf 